\title{
Localization of lattice fermions: lessons for overlap
}

\author{
Benjamin Svetitsky* and Yigal Shamir \\ School of Physics and Astronomy, Raymond and Beverly Sackler Faculty of Exact Sciences, \\ Tel Aviv University, 69978 Tel Aviv, Israel \\ E-mail: bqs@julian.tau.ac.il, shamirepost.tau.ac.il \\ Maarten Golterman \\ Department of Physics and Astronomy, San Francisco State University, San Francisco, \\ CA 94132, USA \\ E-mail: maartenestars.sfsu.edu
}

Lattice fermions in a fluctuating gauge field can show localization, much like electrons in a disordered potential. We study the spectrum of localized and extended states of supercritical Wilson fermions in gauge ensembles generated with plaquette and improved actions. When the Wilson fermion operator is used to construct the overlap kernel, the mobility edge, that is the boundary between the localized and extended states, determines the range of the kernel.

XXIII International Symposium on Lattice Field Theory

25-30 July 2005

Trinity College, Dublin, Ireland

${ }^{*}$ Speaker. 
Localization is a phenomenon long studied in condensed-matter physics [1]. Concerned with conduction in disordered media, it is based on the study of eigenstates of the Schrödinger equation in a random potential. Its counterpart in lattice gauge theory is the study of the spectrum and eigenstates of a Hermitian fermion kernel in the fluctuating gauge field. Here we deal with Wilson fermions, with kernel $H_{W}=\gamma_{5} D_{W}$, in an assortment of gauge ensembles [2,3]. We find low-lying localized states, extending up to an energy called the mobility edge; and extended states at higher energy. We characterize the localized states by their localization length and their support length (related to their inverse participation ratio, or IPR).

The Wilson fermion kernel is not so popular these days for its own sake but rather for its role in constructing domain-wall fermions [4] and the closely related overlap fermions [5]. The simplest overlap kernel is given by

$$
D_{\mathrm{ov}}=1-\gamma_{5} \operatorname{sgn}\left(H_{W}\right) .
$$

The range of $D_{\mathrm{OV}}$ can become long if the spectrum of $H_{W}$ contains low-lying (or zero) modes [6]. We will argue here that, though the energy threshhold of $H_{W}$ lie at zero, the range of $D_{\mathrm{Ov}}$ is determined by the position of the mobility edge - that is, the low-lying modes are generally harmless. The mobility edge perforce lies at non-zero energy, as long as one avoids the Aoki phase [7]. Indeed, the descent of the mobility edge to zero serves as a useful definition of the onset of the Aoki phase.

\section{Localization basics}

As stated above, we address the problem of the spectrum of $H_{W}$ in the random gauge field $U_{x \mu}$. Dislocations in any given configuration $U_{x \mu}$ can create bound states, even zero modes [8], in the spectrum of $H_{W}$. Of course we do not study single configurations of $U_{x \mu}$ but averages over an ensemble. The result is localization.

For a general overview, let's look at Fig. 1. We assume that the free operator $H_{W}$ has a gap for the parameters we choose; above that gap lie plane-wave states. Condensed-matter physicists call the top of the gap the band edge. If we add a single dislocation to the otherwise constant gauge field, the plane waves will be replaced by scattering states and a bound state might appear in the gap. A large number of dislocations will create a large number of bound states, and the scattering states will show effects of multiple scattering. In finite volume, the scattering states as well as the bound states form a discrete spectrum.

In the infinite volume limit, with a fixed density of dislocations, both the bound states and the scattering states form a continuum. The energy that separates the two is called the mobility edge. The infinite volume limit automatically gives us an average over the shapes and positions of the dislocations in any finite subvolume. Alternatively, we can keep the volume finite and average over gauge field configurations ourselves, as is done in lattice gauge theory.

$H_{W}$ can only have zero eigenvalues in the supercritical region, $-8<m_{0}<0$. As it happens, this is just the region of interest for the construction of domain-wall and overlap fermions. The Aoki phase lies in this supercritical region; we stay outside the Aoki phase so that the mobility edge is above zero. We will present numbers [3] below for $m_{0}=-1.5$. For the gauge couplings we choose, the theory lies between the Aoki "fingers." These couplings are in fact popular among users of domain-wall and overlap fermions. 


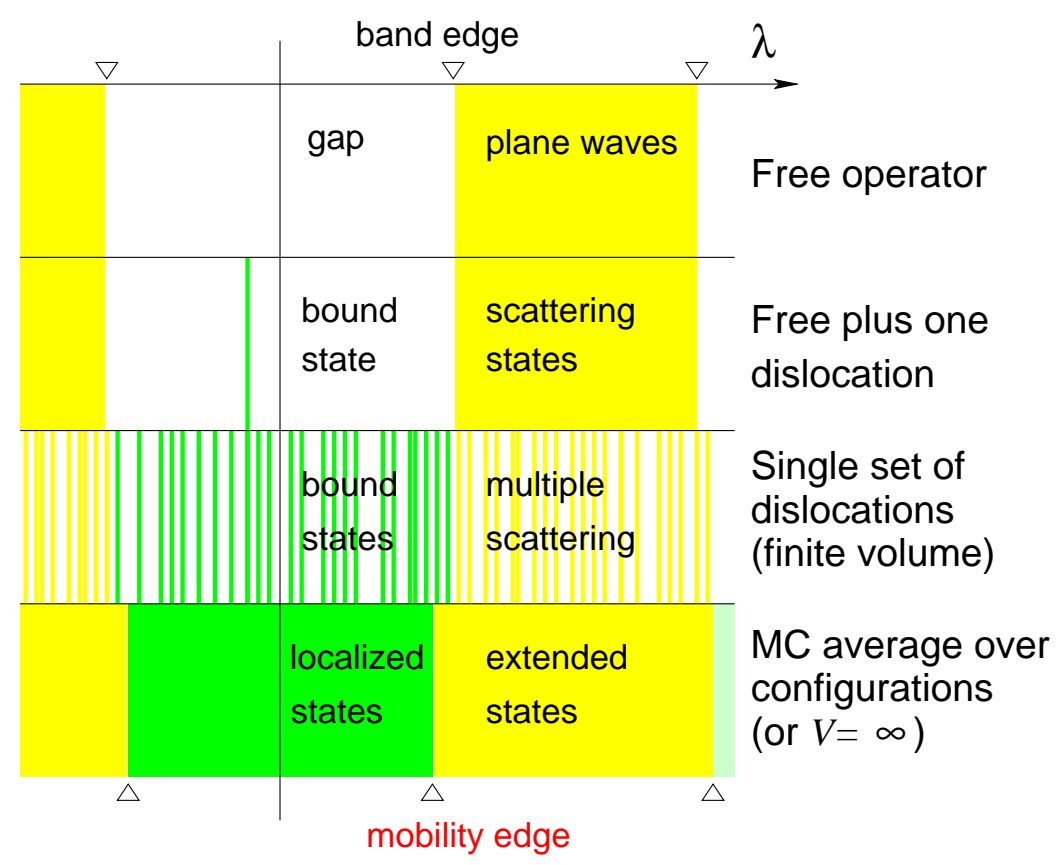

Figure 1: How the spectrum of $H_{W}$ changes as one adds (top to bottom) one dislocation, many dislocations, and a random ensemble of dislocations to the gauge field.

\section{The spectral density}

Since averaging over the gauge field smears the energy eigenvalues, there is never a discrete spectrum of bound states accompanied by a continuum of extended states. The spectrum in the disordered system is described by a continuous density of states,

$$
\rho(\lambda)=\frac{1}{V}\left\langle\sum_{n} \delta\left(\lambda-\lambda_{n}\right)\right\rangle
$$

where $\lambda$ is the energy eigenvalue. We show in Fig. 2 the density of states for the gauge ensemble generated with the Wilson plaquette action. The vertical bars indicate the mobility edge for the two couplings. Nothing special happens at the mobility edge.

[We find it convenient to calculate $\rho$ via the identity

$$
\pi \rho(\lambda)=\frac{1}{V} \lim _{m_{1} \rightarrow 0}\left\langle\operatorname{Im} \operatorname{Tr} G\left(\lambda+i m_{1}\right)\right\rangle
$$

where $G(z)=\left(H_{W}-z\right)^{-1}$ is the resolvent of $H_{W}$. The mobility edge is where the averaged localization length $l_{\ell}(\lambda)$ (see below) diverges.]

The main point of Fig. 2 is to show that there is indeed a nonzero density of states at zero energy. Table 1 shows how this density changes with cutoff and with the choice of gauge action. Improved actions dramatically lower $\rho(0)$, but in no case is it actually zero.

In a theory with dynamical Wilson fermions, these zero modes will be of no consequence because they will cause the fermion determinant to vanish. In any other theory, however, the 


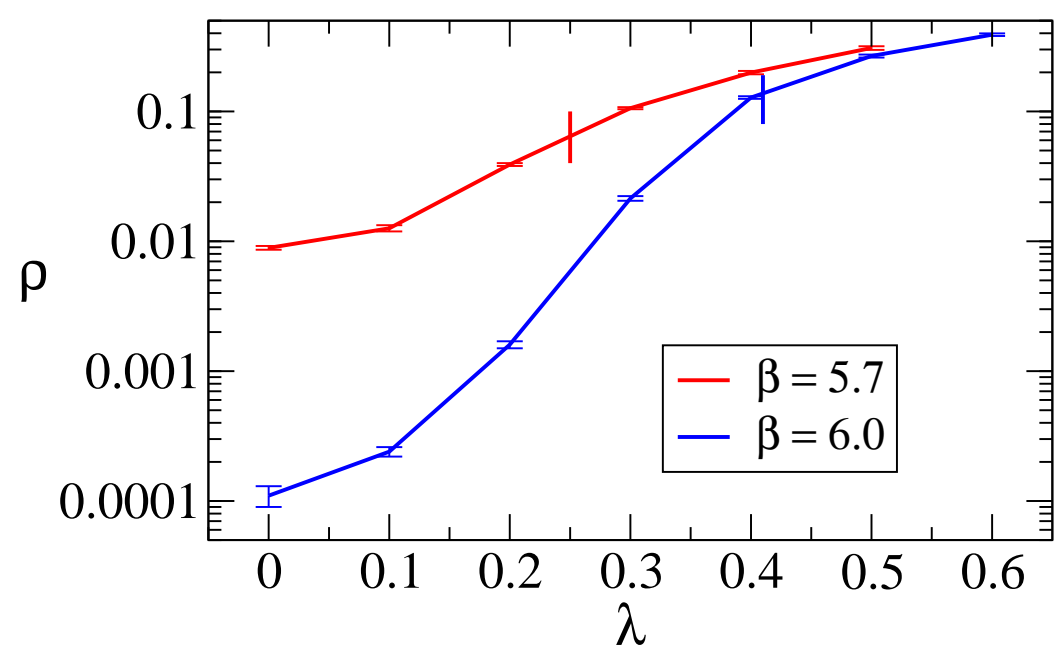

Figure 2: Eigenvalue density $\rho(\lambda)$, in lattice units, for $\beta=5.7$ ( $a^{-1}=1 \mathrm{GeV}$, upper curve) and $\beta=6.0$ $\left(a^{-1}=2 \mathrm{GeV}\right.$, lower curve). Plaquette action.

\begin{tabular}{cccccc} 
& Wilson & & Iwasaki & & DBW2 \\
\hline$a^{-1}=1 \mathrm{GeV}:$ & $\rho(0)=10^{-2}$ & $\Rightarrow$ & $6 \cdot 10^{-3}$ & $\Rightarrow$ & $10^{-3}$ \\
$a^{-1}=2 \mathrm{GeV}:$ & $\rho(0)=10^{-4} \Rightarrow$ & $\Rightarrow$ & $7 \cdot 10^{-7}$ & $\Rightarrow$ & $<10^{-7}$
\end{tabular}

Table 1: Eigenvalue density at $\lambda=0$ for three gauge actions, each at two values of the gauge coupling.

spectrum of $H_{W}$ does not directly determine the fermion determinant. Whether the ensemble is quenched, or whether it contains the domain-wall or overlap fermion determinant, the ensemble average will furnish $H_{W}$ with modes near (and at) zero energy. Will this destroy the locality of $D_{\mathrm{OV}}$ ? No-because (outside the Aoki phase) these modes are localized.

\section{To the overlap kernel}

A localized mode can be described by its support length $l_{s}$, which contains most of the mode's density, and by its localization length $l_{\ell}$, which is the decay length of any long-range tail (see Fig. 3). Each can be averaged over all eigenmodes with eigenvalue $\lambda$, giving $l_{\ell}(\lambda)$ and $l_{s}(\lambda)$. The average support length can be used to determine in what range of $\lambda$ the localized modes are dilute. In view of the mode density shown in Fig. 2, it is clear that low-lying modes will be dilute while the higher modes will be more crowded.

The dilute, the less dilute, and the extended modes all contribute to the overlap kernel $H_{\mathrm{ov}}$. The contribution of the dilute modes, those below some energy $\bar{\lambda}$, is of the form

$$
\begin{aligned}
\left\langle\left|D_{\mathrm{ov}}(x, y)\right|\right\rangle_{\mathrm{loc}} & \approx \int_{-\bar{\lambda}}^{\bar{\lambda}} d \lambda \rho(\lambda) \exp \left(-\frac{|x-y|}{2 l_{\ell}(\lambda)}\right) \\
& \approx \bar{\lambda} \rho(\bar{\lambda}) \exp \left(-\frac{|x-y|}{2 l_{\ell}(\bar{\lambda})}\right),
\end{aligned}
$$




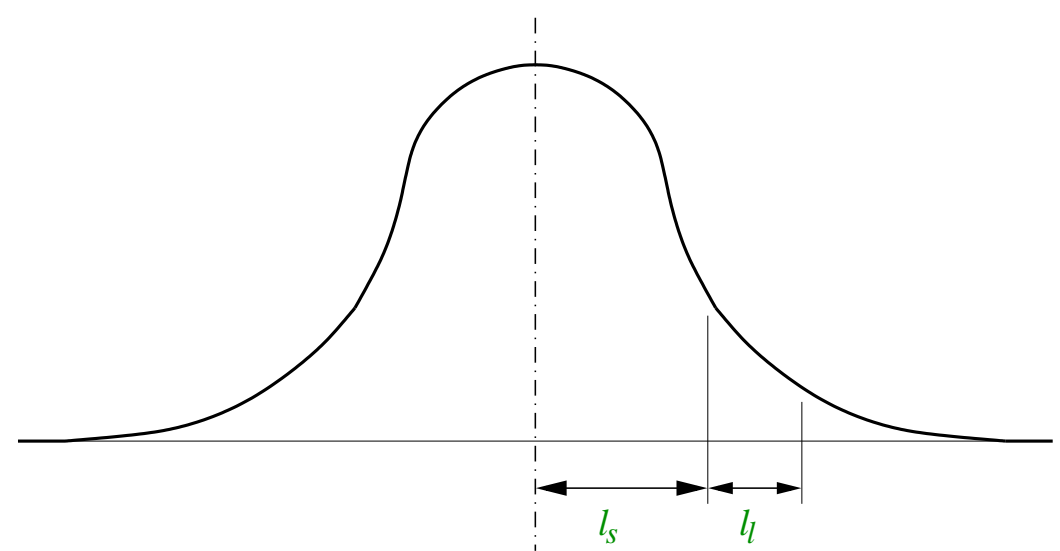

Figure 3: Characteristic lengths of a localized eigenfunction: the support length $1_{s}$, which is the size of the region containing most of the amplitude; and the localization length $l_{\ell}$, which is the decay length of the tail.

where the second line follows from the steep rise in $\rho(\lambda)$ seen in Fig. 2. Note that these isolated modes contribute an exponential tail characterized by their localization length; their low energy is harmless. ${ }^{1}$ The dense modes are up near the mobility edge $\lambda_{c}$; if we lump them with the continuum of extended modes then we can estimate that the fall-off of their contribution will be governed by $\lambda_{c}$, viz.

$$
\left\langle\left|D_{\mathrm{OV}}(x, y)\right|\right\rangle_{\mathrm{ext}} \approx \mathscr{C} \exp \left(-\lambda_{c}|x-y|\right)
$$

where $\mathscr{C}$ is $O(1)$.

Let's put in numbers for the plaquette action at $\beta=6.0$. We measure the mobility edge to be at $\lambda_{c} \simeq 0.41$ and we fix the demarcation point to be $\bar{\lambda} \approx 0.2$. The result is

$$
\left\langle\left|D_{\mathrm{OV}}(x, y)\right|\right\rangle \approx 10^{-4} \exp \left(-\frac{|x-y|}{1.4}\right)+\mathscr{O}(1) \cdot \exp \left(-\frac{|x-y|}{2.4}\right) .
$$

The second term, governed by the mobility edge, wins-both in prefactor and in exponent. We find this to be true in all the cases (actions and couplings) we have studied, including those in Table 1. The reverse could be true in other cases; then the range of $D_{\mathrm{OV}}$ would be determined by the representative localization length, $l_{\ell}(\bar{\lambda})$. The fact that the gap is zero would still be harmless.

We can interpret $\lambda_{c}$ as a mass scale, the mass of effective excitations that influence the overlap kernel. When the cutoff is $a^{-1}=2 \mathrm{GeV}$, we find that $\lambda_{c} a^{-1} \simeq 800 \mathrm{MeV}$ for all three actions. It may be a disappointment to find that the cutoff is felt at an energy that is so low, but this is still well above $\Lambda_{\mathrm{QCD}}$ which is the true dynamical scale. One ought to worry, however, at stronger couplings. When $a^{-1}=1 \mathrm{GeV}$, the value of $\lambda_{c} a^{-1}$ comes out to be only 250-320 MeV, not a place we would like to see unphysical particles in the spectrum.

\section{Conclusions}

The three main lessons of our work:

\footnotetext{
${ }^{1}$ The contribution of dilute localized modes to any Green function will decay in space with the localization length $l_{\ell}$. This is in fact the most convenient way to measure $l_{\ell}(\lambda)$, by calculating almost any Green function [3].
} 
1. The mobility edge at $\lambda_{c}>0$ [or $l_{\ell}(\bar{\lambda})$, if it is greater than $\lambda_{c}^{-1}$ ] assures a finite range for $D_{\mathrm{OV}}$, even though $H_{W}$ has no gap in the disordered gauge field.

2. One should demand that $\lambda_{c} a^{-1} \gg \Lambda_{\mathrm{QCD}}$.

3. $\lambda_{c} a^{-1}$ is fairly insensitive to the gauge action, even as $\rho(0)$ varies widely.

For discussions of these issues in the context of current large-scale lattice computations, see the contributions of T. Draper and of P. Boyle to these proceedings.

\section{Acknowledgments}

This work was supported by the Israel Science Foundation under grant no. 222/02-1, the Basic Research Fund of Tel Aviv University, and the US Department of Energy. Our computer code is based on the public lattice gauge theory code of the MILC collaboration. The code was run on supercomputers of the Israel Inter-University Computation Center and on a Beowulf cluster at San Francisco State University.

\section{References}

[1] D. J. Thouless, Electrons in disordered systems and the theory of localization, Phys. Rep. 13 (1974) 93.

[2] M. Golterman and Y. Shamir, Localization in lattice QCD, Phys. Rev. D 68 (2003) 074501 [hep-lat/0306002]; Localization in lattice QCD (with emphasis on practical implications), Nucl. Phys. Proc. Suppl. 129 (2004) 149 [hep-lat/0309027].

[3] M. Golterman, Y. Shamir and B. Svetitsky, Mobility edge in lattice QCD, Phys. Rev. D 71 (2005) 071502 [hep-lat/0407021]; Localization properties of lattice fermions with plaquette and improved gauge actions, Phys. Rev. D 72 (2005) 034501 [hep-lat/0503037].

[4] D. B. Kaplan, A Method for simulating chiral fermions on the lattice, Phys. Lett. B 288 (1992) 342 [hep-lat/9206013]; Y. Shamir, Chiral fermions from lattice boundaries, Nucl. Phys. B 406 (1993) 90 [hep-lat/9303005]; V. Furman and Y. Shamir, Axial symmetries in lattice QCD with Kaplan fermions, Nucl. Phys. B 439 (1995) 54 [hep-lat/9405004].

[5] R. Narayanan and H. Neuberger, Chiral determinant as an overlap of two vacua, Nucl. Phys. B 412 (1994) 574 [hep-lat/9307006]; H. Neuberger, Exactly massless quarks on the lattice, Phys. Lett. B 417 (1998) 141 [hep-lat/9707022].

[6] P. Hernández, K. Jansen and M. Lüscher, Locality properties of Neuberger's lattice Dirac operator, Nucl. Phys. B 552 (1999) 363 [hep-lat/9808010].

[7] S. Aoki, New phase structure for lattice QCD with Wilson fermions, Phys. Rev. D 30 (1984) 2653.

[8] R. G. Edwards, U. M. Heller and R. Narayanan, Phys. Rev. D 60 (1999) 034502 [hep-lat/9901015]. F. Berruto, R. Narayanan and H. Neuberger, Exact local fermionic zero modes, Phys. Lett. B 489 (2000) 243 [hep-lat/0006030]. 